\title{
Study on the Reduction of Heatsink Radiation by Combining Grounding Pins and Absorbing Materials
}

\author{
Yoeri Arien ${ }^{\# 1}$, Paul Dixon ${ }^{\# 2}$, Mohammad Khorrami ${ }^{\# 2}$ \\ Laird Technologies \\ ${ }^{\# 1}$ Bell Telephonelaan 2B, Geel, Belgium \\ ${ }^{\# 2}$ Randolph, MA, USA \\ Yoeri.arien@lairdtech.com \\ Paul.dixon@lairdtech.com \\ Mohammad.khorrami@lairdtech.com
}

\author{
Andy Degraeve, Davy Pissoort \\ ReMI - Reliability in Mechatronics and ICT \\ Dept. of Electrical Engineering \\ KU Leuven - Technology Campus Ostend \\ Zeedijk 101, B8400, Ostend, Belgium \\ andy.degraeve@kuleuven.be \\ davy.pissoort@kuleuven.be
}

\begin{abstract}
This paper describes a study on the effectiveness of grounding pins and high-frequency absorbers to reduce the emissions of heatsinks up to $20 \mathrm{GHz}$. The study considers four different types of excitations, namely a direct excitation, an excitation by a patch antenna, an excitation by a microstrip below the center of the heatsink, and an excitation by a microstrip that was off-set with respect to the center of the heatsink. Grounding post are positioned equidistantly along the edges of the heatsink and the number of grounding pins per side is varied. Adding grounding pins significantly reduces the emission of the heatsink below 1-2 GHz. At higher frequencies, they lose their capability to reduce the heatsink emission and even introduce extra resonance frequencies where the emission is higher compared to the ungrounded heatsink. Three different possible arrangements for the application of absorbing material were considered, namely a thin flat absorber just below the heatsink, a ring absorber between the heatsink and the PCB, and a collar absorber around the heatsink. The absorber succeeds in reducing the heatsink's emission above 1-2 GHz, but has little of no positive effect on the emissions below 1-2 GHz. Only by the combination of the grounding pins and the absorber material, a reduction of the heatsink's emission is achieved over the full frequency range.
\end{abstract}

Keywords-heatsink radiation, high-frequency grounding, high-frequency absorbers

\section{INTRODUCTION}

The continuing increasing complexity and integration density of Integrated Circuits (ICs) inevitably leads to the creation of a significant amount of heat. As a results, heatsinks are very frequently applied to maintain an acceptable IC temperature. Unfortunately, heatsinks are typically made of metals that have both a high thermal and electrical conductivity. Due to the combination of that high electrical conductivity and the increasing clock frequencies of the ICs, the heatsink easily becomes a very efficient antenna over a large frequency range.

In literature, several papers can be found that discuss techniques to reduce a heatsink's emission. Most papers propose to use appropriate high-frequency grounding of the heatsink, see e.g [1][2] and the references therein. A general conclusion that can be drawn from these papers is that more grounding pins means less radiation. However, to be very effective, the location of the grounding pins should be optimized. Unfortunately, this is not always possible in a real application where the IC might take quite a large space underneath the heatsink and, hence, only grounding pins along the edge of the heatsink are possible. A few papers propose the use of high-frequency absorbing materials [3]. However, little information can be found on the dependency of the efficiency of such an absorber on the way it is mounted to the heatsink. In most studies, the heatsink is excited by a voltage source between the ground-plane on the Printed Circuit Board (PCB) and the heatsink. Little information can be found on how well this excitation represents a typical IC.

In this paper, full-wave FDTD simulations are used to study the effectiveness that (i) grounding pins, (ii) absorbing materials, and (iii) the combination of grounding pins and absorbing materials have on a heatsink's emission. In [4], it has been shown such full-wave simulations correlate very well with measurements. Contrary to most previous papers, four different types of excitations are considered. Also for the absorbing material, three different arrangements are considered.

This paper is organized as follows. Section II describes the general set-up of the heatsink-under-study as well as the different excitations that are applied. Section III discusses the influence of grounding pins on the heatsink's emission. Section IV discusses the influence of absorbing materials on the heatsink's emission. Section V shows that by combining grounding pins and absorbing materials a significant reduction of the heatsink's emission is obtained over the whole frequency range. Section VI draws concluding remarks.

\section{INFLUENCE HEATSINK EXCITATION}

\section{A. Description set-up}

For simplicity, the heatsink has been modeled as a PEC block with dimension of (W x L x H) 40 mm x 40 mm x 10 $\mathrm{mm}$. The heatsink is mounted $2 \mathrm{~mm}$ above a two layer FR4 PCB of (W x L x H) $80 \mathrm{~mm} \times 160 \mathrm{~mm} \times 1.5 \mathrm{~mm}$. The bottom layer of the PCB comprises a full PEC ground plane. The total structure is shown in Fig. 1. Previous 
investigations have shown that including the fins in the heatsink model did not affect the results.

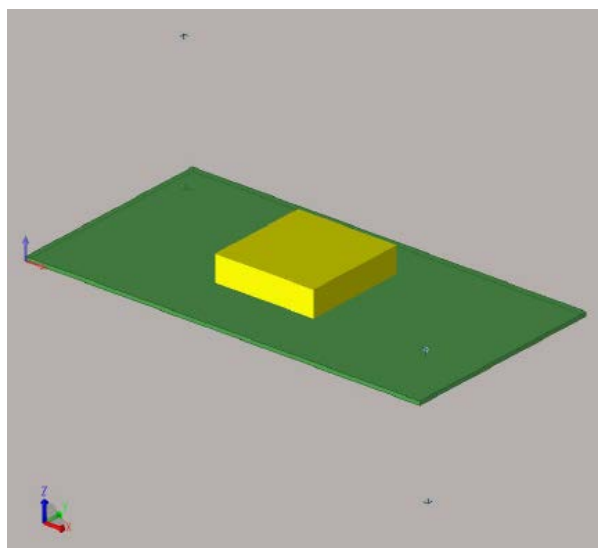

Fig. 1. Heatsink-under-test

Figure 2 depicts the four different excitations that are used:

1. A direct excitation by means of a vertical 1 Volt 50 Ohm source connected between the center of the ground plane and the center of the heatsink's bottom side.

2. An excitation by a patch antenna (fed by a 1 Volt $50 \mathrm{Ohm}$ source) of $20 \mathrm{~mm}$ by $20 \mathrm{~mm}$, centered below the heatsink.

3. An excitation by a microstrip of $20 \mathrm{~mm}$ by $2 \mathrm{~mm}$, centered below the heatsink. The microstrip is fed by a 1 Volt - 50 Ohm source at one end and terminated in $50 \mathrm{Ohm}$ at the other end.

4. An excitation by a similar microstrip, but now offcentered by $8 \mathrm{~mm}$ and $12 \mathrm{~mm}$ in the $\mathrm{X}$ and $\mathrm{Y}$ direction, respectively.
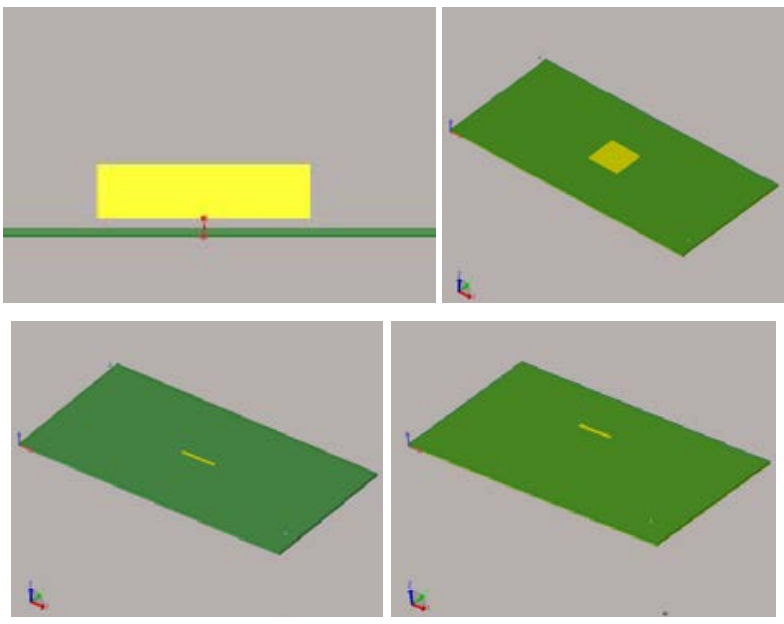

Fig. 2. Different excitations (top left: direct; top right: patch; bottom left: microstrip, centered; bottom right: microstrip, off-set)

The total radiated power of the ungrounded heatsink is shown in Fig. 3 for these four different excitations from 10 $\mathrm{MHz}$ up to $20 \mathrm{GHz}$. The radiated power has been obtained with the FDTD solver included in Keysight Technologies’
3D simulation tool EMPro [5]. The direct excitation leads to the worst-case emission of the largest part of the frequency range. However, the other types of excitations give rise to more pronounced resonances.

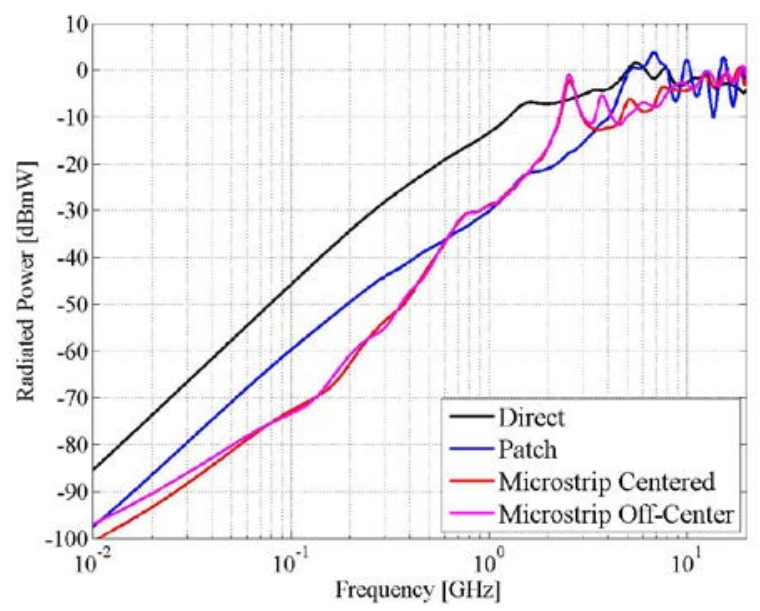

Fig. 3. Radiated power, ungrounded heatsink, no absorber

\section{REDUCTION OF RADIATION WITH GROUNDING PINS}

A common method to reduce a heatsink's emissons is to apply grounding pins. Although previous studies indicate that the number and locations of these grounding pins must be optimized [1][2], in practice these grounding pins are typically distributed equidistantly along the edges of the heatsink (Fig. 4).

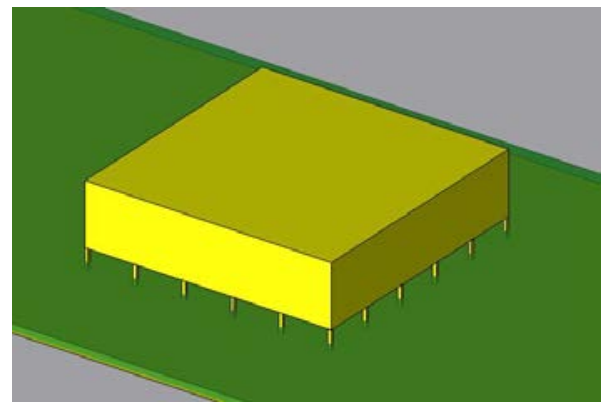

Fig. 4. Grounding pins heatsink

Figures 5-8 show the radiated power of the grounded heatsink for an increasing number of grounding pins per side and this for the four different excitations mentioned above. In all cases, the grounding pins reduce the emissions of the heatsink up to a frequency of $1-2 \mathrm{GHz}$. However, around these frequencies an extra LC resonance is introduced because of the interaction between the inductance of the grounding pins and the capacitance between the heatsink and the PCB [1]. Increasing the number of grounding pins shifts this resonance to a higher frequencie because of the lower total inductance of the grounding pins. Unfortunately, a large number of pins has to 
be used to get any reduction of the heatsink's emission above a few GHz.

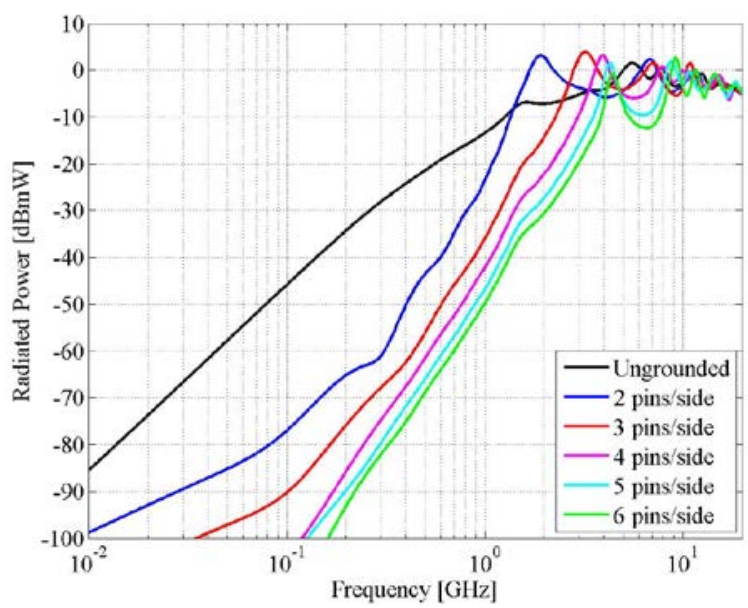

Fig. 5. Radiated power heatsink vs number of grounding pins, no absorber, direct excitation

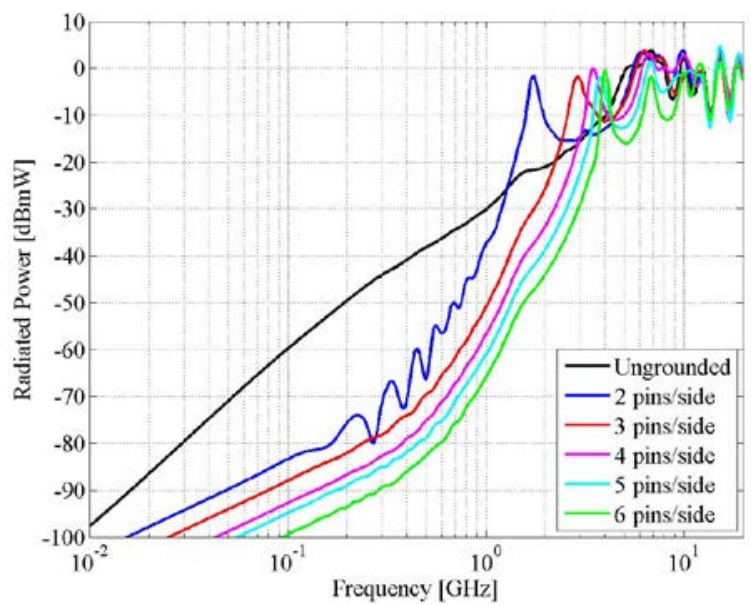

Fig. 6. Radiated power heatsink vs number of grounding pins, no absorber, patch excitation

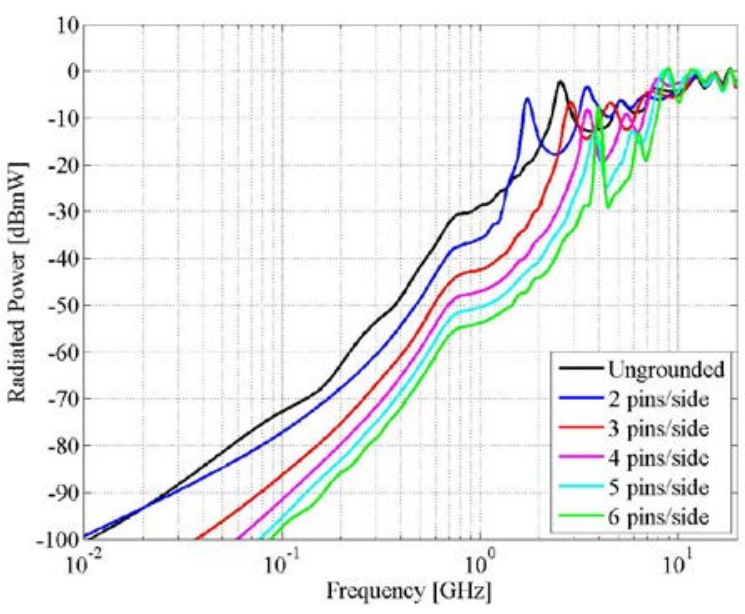

Fig. 7. Radiated power heatsink vs number of grounding pins, no absorber, excitation by centered microstrip

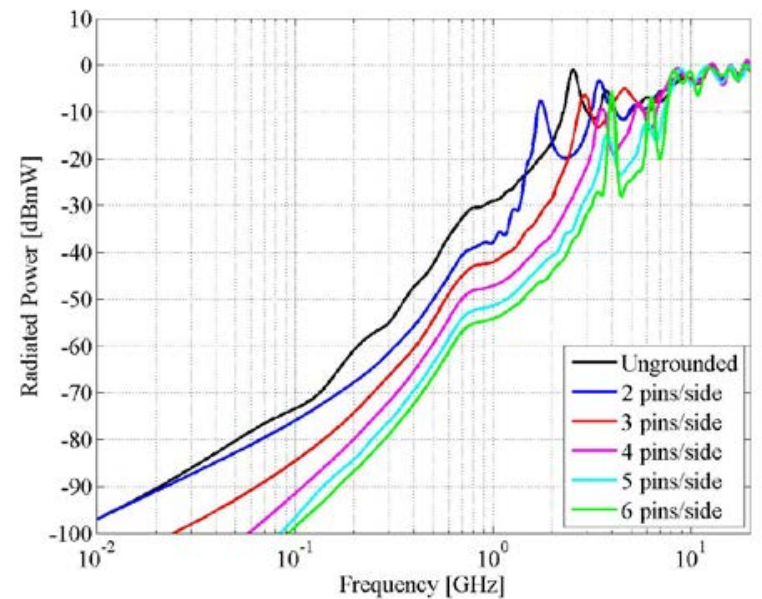

Fig. 8. Radiated power heatsink vs number of grounding pins, no absorber, excitation by off-set microstrip

\section{REDUCTION OF RADIATION WITH ABSORBERS}

An alternative method to reduce a heatsink's emission is to apply a lossy, high-frequency absorber. In this section, the influence of the ECCOSORB BSR-1 absorber is investigated. This ECCOSORB BSR-1 absorber is modelled in EMPro as a combination of 3-pole electric Debye model and a 1-pole magnetic Debye model. Material parameters are obtained as described in [4].
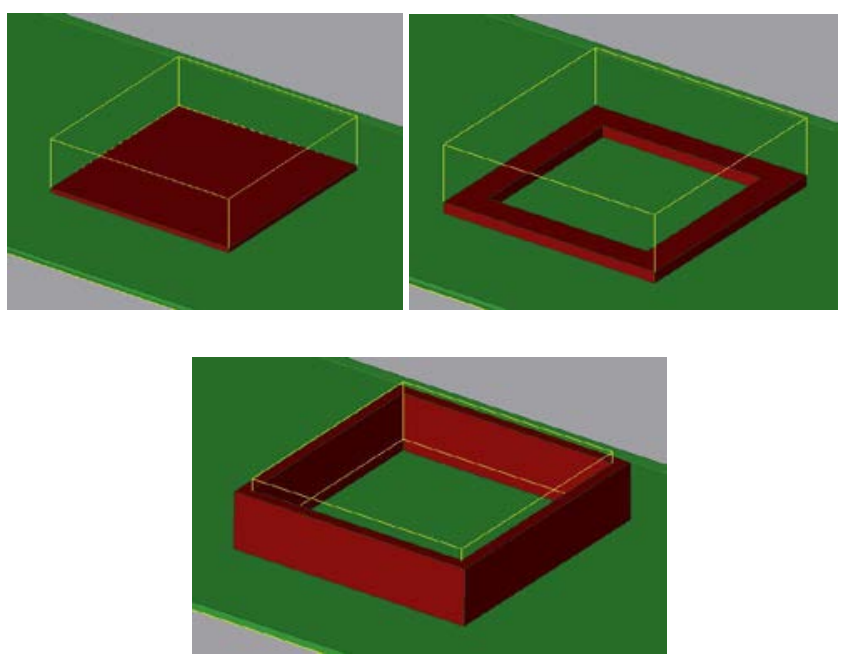

Fig. 9. Positioning absorbers (top left: flat absorber; top right: ring absorber; bottom: collar absorber)

In most previous studies, a flat thin sheet of absorber is attached to the bottom side of the heatsink. However, this is not always the preferred way in practice because of the relatively poor thermal properties of most absorbers. Therefore, Fig. 9 proposes two alternative arrangements for the absorber, namely a ring of absorbing material at the edge 
of the heatsink or a collar of absorbing material around the heatsink.

Figures 10-13 show the radiated power of the ungrounded heatsink for the different arrangments of the absorber and this for the four different excitations mentioned above. In all cases, the absorber reduces the emissions of the heatsink above the frequency of 1-2 GHz. Below that frequency, the absorber has either only little influenc on the emission or even increases it. Note that the exact behavior of the absorber depends on the type of excitation. It is known that the effectiveness of an absorbers depends heavily on the exact field distribution inside the "cavity" into which it is placed. Different excitation types mean different field distributions and, hence, a different absorber performance.

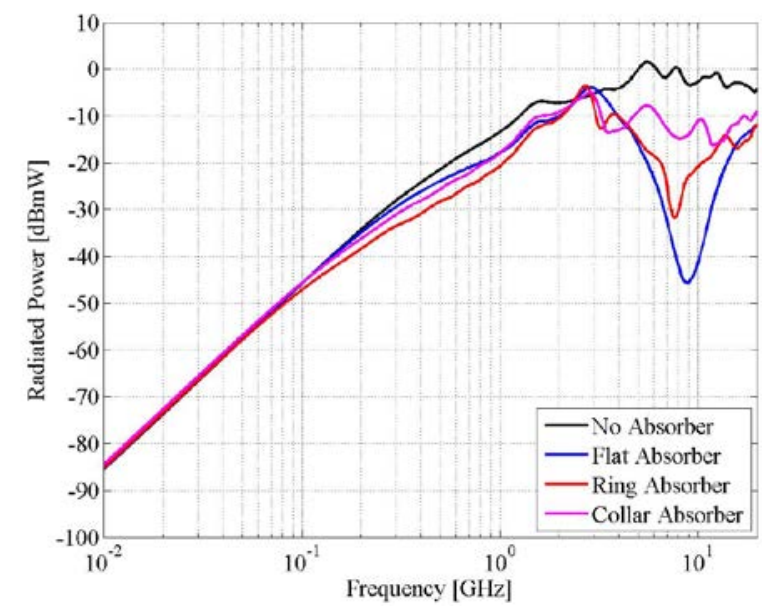

Fig. 10. Radiated power ungrounded heatsink with ECCOSORB BSR-1 absorber, direct excitation

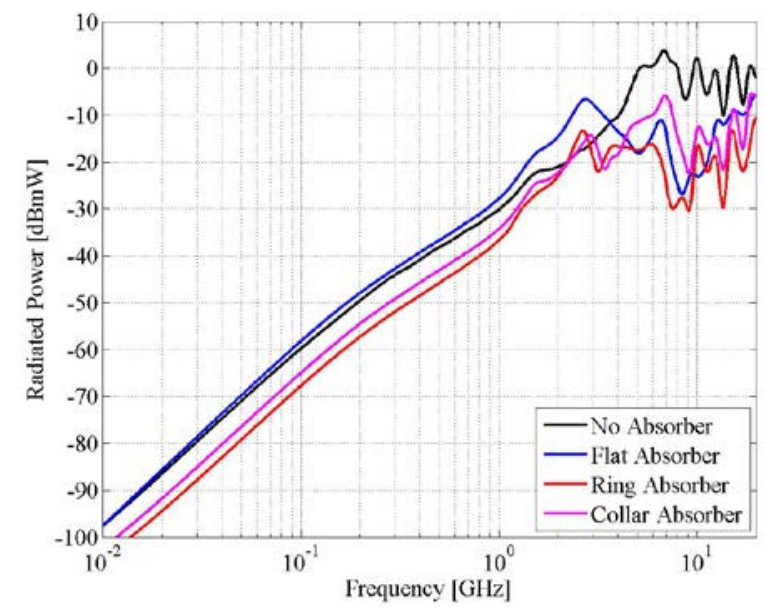

Fig. 11. Radiated power ungrounded heatsink with ECCOSORB BSR-1 absorber, patch excitation

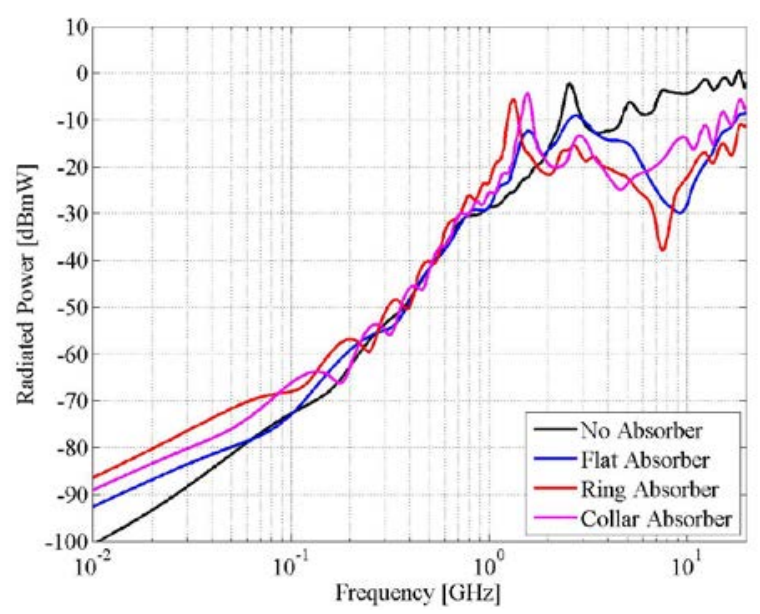

Fig. 12. Radiated power ungrounded heatsink with ECCOSORB BSR-1 absorber, excitation by centered microstrip

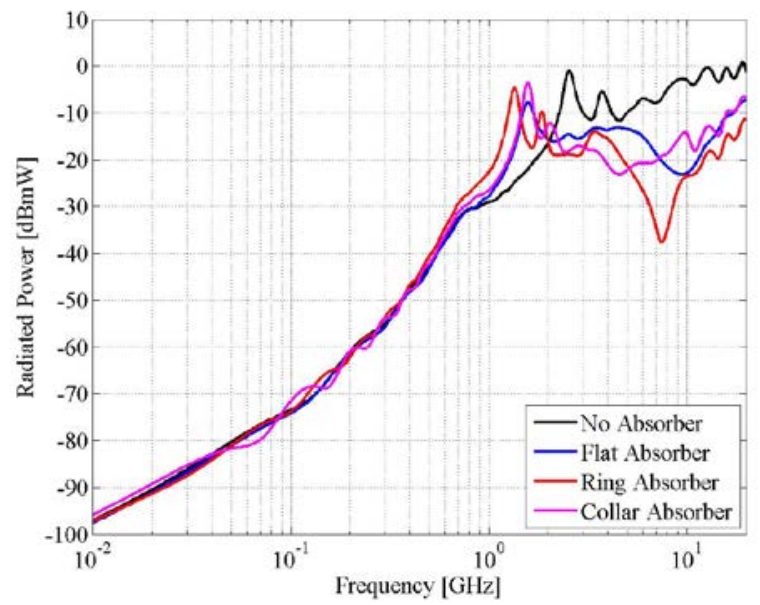

Fig. 13. Radiated power ungrounded heatsink with ECCOSORB BSR-1 absorber, excitation with off-set microstrip

\section{COMBINING GROUNDING PINS AND ABSORBERS}

Based on the conclusions from Section III and IV, the optimal solution might be to combine grounding pins and a high-frequency absorber. Figures 14-17 show the radiated power for a heatsink grounded with 6 pins/side in combination with a high-frequency absorber. Again, the three different arrangements for the absorber as well as the four excitation types are considered. In all cases, a good reduction of the heatsink's emission is obtained over nearly the whole frequency range. Again, the absorber's performance depends on the exact nature of the exciting source. 


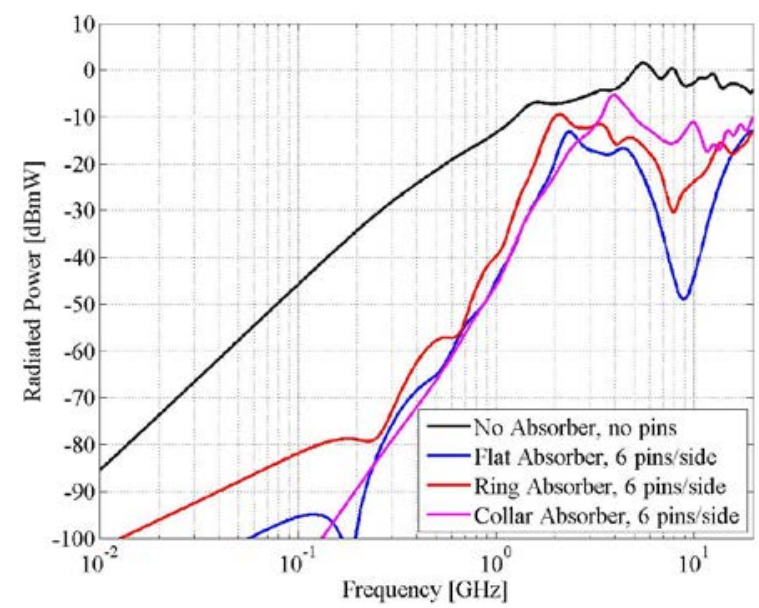

Fig. 14. Radiated power grounded heatsink (6 pins/side) with ECCOSORB BSR-1 absorber, direct excitation

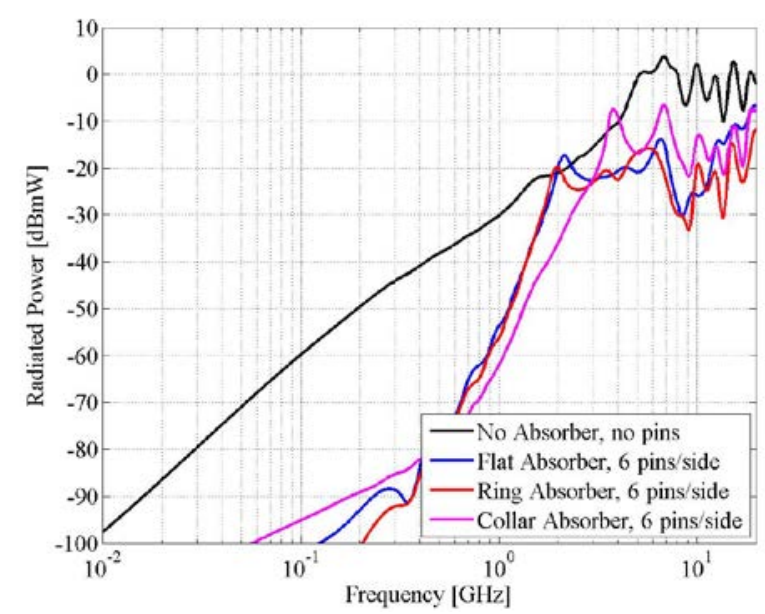

Fig. 15. Radiated power grounded heatsink (6 pins/side) with ECCOSORB BSR-1 absorber, patch excitation

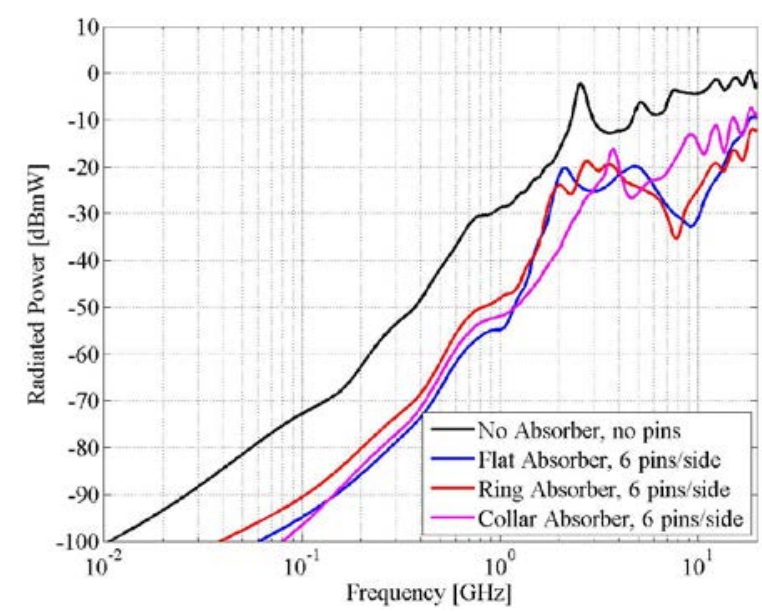

Fig. 16. Radiated power grounded heatsink (6 pins/side) with ECCOSORB BSR-1 absorber, excitation with centered microstrip

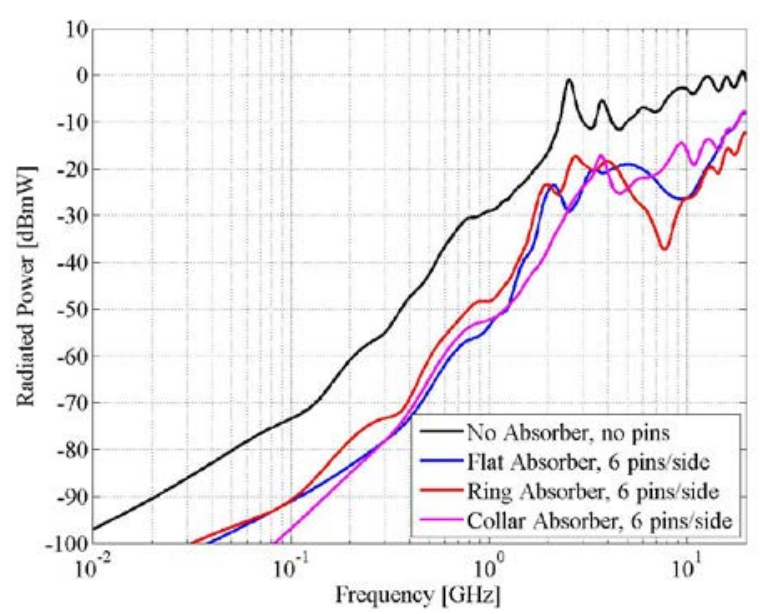

Fig. 17. Radiated power grounded heatsink (6 pins/side) with ECCOSORB BSR-1 absorber, excitation with off-set microstrip

\section{CONCLUSIONS}

By means of full-wave simulations, the effectiveness of grounding pins and high-frequency absorbers to reduce heatsinks' emissions was studied in the frequency range from $10 \mathrm{MHz}$ up to $20 \mathrm{GHz}$. Four different excitation types were considered. By increasing the number of grounding pins along the edges, the emission of the heatsink is significantly reduced up to 1-2 GHz. However, additional LC resonances are introduced and the grounding pins lose their effectiveness at higher frequencies. By application of absorbing materials, the heatsink's emissions above $1-2 \mathrm{GHz}$ can be reduced. The level of reduction depends on the way that the absorber is mounted to the heatsink. Below 1-2 GHz, the absorber seems to have little influence on the heatsink's emission. By combining the grounding pins with the absorbing material, the heatsink's emission can be reduced of the whole frequency range.

\section{ACKNOWLEDGMENT}

This research was supported by the IWT (Flanders, Belgium) under the EMCAD project.

\section{REFERENCES}

[1] Xinbo He, T Hubing, " Mitigation of Unintentional Radiated Emissions from Tall VLSI Heatsinks Using Grounding Posts”, IEEE Transactions on EMC, vol. 55, no. 6, Dec. 2013, pp. 1271-1276

[2] Archambeault, B.; Pratapneni, S.; Zhang, L.; Wittwer, D.C., "Comparison of various numerical modeling tools against a standard problem concerning heat sink emissions," Electromagnetic Compatibility, 2001. EMC. 2001 IEEE International Symposium on , vol.2, no., pp.1341,1346 vol.2, 2001

[3] Jim Chiappe, "Additional Techniques to Reduce Heatsink Emissions Utilizing RF Absorbers", Proceedings of the IEEE International Conference on Electromagnetic Compatibility, Pittsburgh, PA, USA, August 2012, pp. 53-63

[4] Qian Liu, Xiangyang Jiao, Jing Li, Victor Khilkevich, James Drewniak, Paul Dixon and Yoeri Arien, "Modeling absorbing 
materials for EMI mitigation", Submitted to IEEE International Conference on Electromagnetic Compatibility, Santa Clara,

California, USA, March 2015
[5] Keysight Technologies, http://www.keysight.com/
EMPro, available from: 